\title{
A Lagrangian approach for modelling electro-kinetic mass transfer in microchannels
}

\author{
Jahrul M Alam, ${ }^{\mathrm{a}, *}$ Jared M Penney ${ }^{\mathrm{a}, 1}$ \\ ${ }^{a}$ Department of Mathematics and Statistics, Memorial University, Canada, A1C 5S7
}

\begin{abstract}
The computational modelling of multiphysics mass transfer in a microchannel is a challenging endeavour. This article has proposed and validated a Lagrangian methodology for modelling coupled physics using near optimal computational efforts. This new development has been verified and compared with a reference Eulerian finite difference model. A heuristic theory for computational mass transfer phenomena has been studied. At high Peclet numbers when the reference Eulerian model fails, the Lagrangian model resolves electro-osmotic mass transfer, showing a good quantitative agreement with theoretical analysis. The Lagrangian model also helps to estimate necessary parameters so that an optimal electro-osmotic pumping can be designed in a microchannel. The computational efficiency of the Lagrangian model has been examined, showing that an increase of the Peclet number by a factor of 32 increases the global computational complexity by about a factor of $10^{4}$ if the reference Eulerian model were used. This verifies the optimal performance of the Lagrangian model.
\end{abstract}

Keywords: mass transfer; electro-kinetic; Lagrangian; electro-osmosis;

\footnotetext{
*Corresponding author

Email address: alamj@mun . ca (Jahrul M Alam)

${ }^{1}$ Present address: Department of Applied Mathematics, University of Waterloo, Canada, N2L $3 \mathrm{G} 1$
} 
multigrid.

\begin{tabular}{|c|c|c|c|}
\hline \multicolumn{4}{|c|}{ NOMENCLATURE } \\
\hline$v$ & kinematic viscosity & $\boldsymbol{u}$ & velocity vector \\
\hline$\rho$ & density & $P$ & pressure \\
\hline$\psi$ & wall potential & $f$ & force \\
\hline$\varphi$ & external potential & $t$ & time \\
\hline$\phi$ & scale for external potential & $U$ & velocity scale \\
\hline$\zeta$ & scale for wall potential & $h$ & channel width \\
\hline$\alpha$ & forcing parameter & $H$ & channel length \\
\hline$\eta$ & electrophoretic mobility & $\nabla$ & gradient \\
\hline $\mathcal{B}$ & Boltzmann constant & $\nabla^{2}$ & Laplacian \\
\hline$e$ & elementary charge & $\Gamma$ & concentration flux \\
\hline$T$ & absolute temperature & $C$ & concentration \\
\hline$\epsilon$ & permittivity & $D$ & diffusion coefficient \\
\hline$n$ & ion density & $\Delta x, \Delta y$ & grid spacing \\
\hline $\mathcal{A}$ & Avogadro's number & $\Delta t$ & time step \\
\hline $\mathcal{D}$ & Debye layer thickness & $n_{x} \times n_{y}$ & resolution \\
\hline$\xi$ & streamline parameter & $\mathcal{L}$ & general partial differential operator \\
\hline $\operatorname{Re}$ & Reynolds number & $P e$ & Peclet number \\
\hline \multicolumn{4}{|c|}{ Superscripts } \\
\hline$n$ & time step & $n+1 / 2$ & fractional time step \\
\hline \multicolumn{4}{|c|}{ Subscripts } \\
\hline$i, j$ & grid point & $\boldsymbol{k}$ & index for streamline \\
\hline
\end{tabular}




\section{Introduction}

The study, design, and analysis of microfluidics and nanofluidics are widespread in many scientific and industrial contexts [1-4]. For example, understanding the electro-osmotic micro-dynamics is important in various microfluidic systems, such as microelectromechanical systems (MEMS), biological microelectromechanical systems (BioMEMS), and lab-on-a-chip [5-7]. A computational model - solving the mass and momentum conservation laws numerically - is a virtual experimentation of such micro-dynamics, and has the potential to advance the fundamental knowledge of physical, chemical, and biological processes in areas such as micro-fabrication, biotechnology, and portable devices. There is a growing interest in using computational models in microfluidic research. This article presents a novel computational model for simulating electro-osmotic mass transfer in a microfluidic device.

In the past two decades, a number of articles have been published studying the usefulness of computational models for simulating electro-kinetics in microfluidic devices. Among the pioneering works, Patankar and $\mathrm{Hu}$ [8] studied the development of a steady state three-dimensional numerical model for simulating electro-osmotic flow in a cross-channel microfluidic device. In their work, a finite volume approach using the SIMPLE class of pressure solver was adopted to computationally imitate the experimental investigation of Harrison et al. [9]. As of the writing of this article, the computational model of Patankar and $\mathrm{Hu}$ [8] has received over 400 citations, and has provided guidelines to further computational developments for scientists and engineers who have interests in computational modelling of electro-kinetic phenomena [e.g. 10-14]. Over the years, finite difference (FD) [15-17], finite volume (FV) [8, 18], and finite element (FE) [10, 19, 20] 
methods have been commonly used by researchers investigating a fluid flow in microfluidic devices [21]. Other methods such as the level set method [22], Lagrangian particle methods [23] or higher order weighted/essentially non-oscillatory (W/ENO) [24] schemes are not fully examined in the field of microfluidic modelling [e.g. 25]. A brief review of recent literature indicates that the best numerical algorithm for simulating flows in microfluidic devices is not fully understood, and significant improvements in two directions are necessary for taking full advantage of advanced computational methods in the field of microfluidic research. First, multilevel and multiresolution methods [26] may resolve more economically the electric double layer and the moving sharp interface between the transported fluid and the resident fluid. Second, fully capturing the coupled multiphysics nature of the electro-osmotic mass transfer would provide sophisticated modelling strategies. The drawbacks of classical numerical modelling in this direction - particularly for mass transfer in micro-fluidic devices - are not fully addressed by recent computational micro-fluidic models. Current authors are interested in combining the benefits of multiple advanced computational methodologies - such as the Lagrangian method and the multigrid/multilevel solver - into integrated modelling frameworks to address the latter of the above issues. This facilitates the development of simpler and less expensive methodologies for modelling mass transfer problems that involve more than one physical phenomenon.

Electro-hydrodynamics or electro-kinetic phenomena often refers to a combination of electro-osmotic and electrophoretic transport [27, 28]. The topic is a scientific problem of multiphysics modelling, where at least three physical phenomena - electrostatics, mass/momentum conservation, and ion transport - must be modelled simultaneously. A computational model would resolve these physics 
by solving a simultaneously coupled system of governing equations that include the incompressible Navier-Stokes equation, the Poisson-Boltzmann equation, the Laplace equation, and the Nernst-Planck equation. Computational modelling of each of these physics requires careful attention to designing sophisticated algorithms. First, the incompressible Navier-Stokes equation couples the velocity and pressure, which requires special treatment [e.g. 29]. A multilevel method is examined in this article to address this problem. Second, the Nernst-Planck equation takes an almost hyperbolic form because the transport of ions is principally governed by inertial effects [ch. 11, 30]. This article proposes the use of a Lagrangian methodology and examines the potential of this technique. Here, we note that a classical computational problem of mass and momentum transfer will often aim to improve the stability of a scheme, the order of numerical accuracy, and the speed of computation, but a computational multiphysics modelling approach will aim for a balance between numerical speed/accuracy, and the quality of coupled physics. Hence, a successful computational model of electro-kinetic and/or hydrodynamic transport in a micro-device remains challenging. One goal of the present article is to identify the source of computational error that destroys the quality of the coupled physics, and to postulate a modelling framework so that the need for computing speed and accuracy does not sacrifice the quality of coupled physics.

This article introduces a streamline-based Lagrangian computational model for the numerical simulation of electro-kinetically driven transport phenomena in microfluidic devices. To the best of authors' knowledge, none of reported computational developments use such a streamline-based Lagrangian approach. Note that instantaneous velocity vectors are tangential to streamlines, and these streamlines do not start or end inside the fluid region. Clearly, streamlines are more 
relevant to the physics of the flow than randomly seeded finite number of particles those are commonly traced in classical Lagrangian models. A two- or threedimensional transport law can therefore be reduced to a one-dimensional transport problem in the streamline coordinate. This one-dimensional problem can be solved analytically, improving the modelling quality and saving the computing time. The streamline based Lagrangian approach provides a natural framework for modelling transport phenomena, where a finite number of computed streamlines model the time evolution of electro-kinetic transport at high Peclet numbers.

Principal investigations of this article include the following. First, we want to study on the benefits of Lagrangian modelling approach for simulating microscale flows, and introduce a streamline-based Lagrangian method. Second, we want to extend the scope of the heuristic stability analysis method [31], showing how to identify source of computing error in microscale multiphysics simulations. Third, we want to explore the combined benefits of multilevel and Lagrangian methods so that the quality of the solution is improved with optimal computational efforts.

In Section 2, the governing equations and the proposed modelling approach are presented. In Section 3, we have studied the potential for such a Lagrangian approach to model a micro-scale flow, and compared its performance with respect to a classical approach. We have also studied a heuristic theory for computational mass transfer phenomena. In Section 4, results of numerical experiments are discussed. In Section 5, a brief summary, present contributions, and potential future research directions are outlined. 


\section{The streamline based Lagrangian modelling}

\subsection{Governing equations}

In 1976, Batchelor [32] introduced the notion of microhydrodynamics for modelling fluid flows with characteristic length scales at the order of microns $(1 \mu \mathrm{m}$ $\left.=10^{-6} \mathrm{~m}\right)$. This idea remains at the core of multiphysics mass transfer modelling at micro-scale, despite many other attempts [33]. Thus, the governing equations for a micro-scale electro-osmotic flow can be derived from the fundamental principles studied earlier by Debye and Hückel [34], Batchelor [32], and Helmholtz [35]. Assuming that the fluid with suspended charged particles is Newtonian and incompressible, the mass and momentum conservation laws take the dimensionless forms

$$
\begin{gathered}
\boldsymbol{\nabla} \cdot \boldsymbol{u}=0, \\
\frac{\partial \boldsymbol{u}}{\partial t}+\boldsymbol{u} \cdot \boldsymbol{\nabla} \boldsymbol{u}=-\boldsymbol{\nabla} P+\frac{1}{\operatorname{Re}} \nabla^{2} \boldsymbol{u}+\boldsymbol{f},
\end{gathered}
$$

where the Reynolds number is defined by $R e=\frac{U h}{v}$. The length $(h)$ and velocity $(U)$ scales are listed in Table 2. For a list of dimensional parameters, such as $v$, see Table 1 . The force $f$ is a result of the combined effect of an externally applied electric field and the potential due to the charge at the device boundary that are exerted simultaneously on a fluid with a large number of suspended species [e.g. see 8]. As explained by Batchelor [32], a Gibbs distribution for the anion of the form, $n \mathcal{A} \exp \left(\frac{e \psi}{\mathcal{B} T}\right)$, can be used to model the effect of the wall potential. Taking the charge density as the sum of charge densities for both the cations and the anions,

and using the Debye-Hückel approximation, $\mathcal{D}^{2}=\frac{\mathcal{B} T \epsilon}{e^{2} n \mathcal{A}}$, we have the following Helmholtz equation for the wall potential $\psi$,

$$
\nabla^{2} \psi=\kappa^{2} \psi
$$


where $\kappa^{2}=\frac{h^{2}}{\mathcal{D}^{2}}$. Accordingly, the externally applied potential satisfies the Laplace's equation

$$
\nabla^{2} \varphi=0
$$

Hence, the electro-osmotic force, $f$, is given by [e.g. 8, 16]

$$
f=\alpha \psi \nabla \varphi
$$

where

$$
\alpha=\frac{\zeta \phi \epsilon}{\rho U^{2} \mathcal{D}^{2}} .
$$

Letting the concentration of the passive suspended species be $C(\boldsymbol{x}, t)$, the flux $\boldsymbol{\Gamma}$ of the species can be given by $\boldsymbol{\Gamma}=\boldsymbol{u} C-(\eta \nabla \varphi) C$, which accounts for the hydrodynamic flux, $\boldsymbol{u} C$, and the electrophoretic flux, $(\eta \nabla \varphi) C$ [36]. The concentration satisfies the following form of the dimensionless Nernst-Planck equation [ch.11, 30]

$$
\frac{\partial C}{\partial t}+\nabla \cdot(\boldsymbol{u}-\eta \nabla \varphi) C=\frac{1}{P e} \nabla^{2} C
$$

where we have assumed that the derivatives of $\psi$ are negligible compared to that of $\varphi$ in regions far from the boundary of the device, and dimensionless electrophoretic mobility is $\eta=\frac{\tilde{\mu} \phi}{U h}$. Note the Peclet number is defined by

$$
P e=\frac{U h}{D},
$$

where a large $P e$ corresponds to a small diffusion coefficient $D$. The governing equations (1-5) model an electro-kinetic flow in a microchannel that can be driven by an electric force or a pressure drop, where all dimensional parameters and scales - as listed in Tables 1, 2 - have been combined into four dimensionless quantities: $R e, P e, \alpha$, and $\kappa$. 


\subsection{Geometry and boundary conditions}

To solve the set of model equations (1-5) numerically, a rectangular channel of width $h$ and length $H$ with walls along the $x$-axis is used in the present development. No-slip and Neumann boundary conditions are assumed for the velocity at walls and the input/output boundaries resepectively. For the pressure and concentration, boundary conditions are Neumann at the walls and Dirichlet at the input and output.

\subsection{Numerical method}

All spatial derivatives in Eqs (1-5) have been computed with a second order accuracy. An implicit-explicit (IMEX) method is used to solve (2), where the viscous forces are treated implicitly using a second-order Crank-Nicolson method. With the help of Eq. (1), the nonlinear inertial terms in (2) are expressed in their flux form, and treated with the explicit Euler method. A fractional time integration method has been employed to Eq. (5) so that the mass transport can be modelled with the proposed Lagrangian method, and mass diffusion can be solved with the Crank-Nicolson method.

The electro-osmotic force $f$ is computed by solving Eqs (3-4) before starting the time integration. At each time step, the pressure is computed by solving the Poisson equation

$$
\nabla^{2}\left(P+\frac{\boldsymbol{u} \cdot \boldsymbol{u}}{2}\right)=\boldsymbol{\nabla} \cdot[\boldsymbol{f}+\boldsymbol{u} \times(\boldsymbol{\nabla} \times \boldsymbol{u})] .
$$

Eqs $(3,4,6)$ and two other Crank-Nicolson systems are solved using a multigrid algorithm [16]. A distinct feature of the present work includes the discretization of (6) employing a modified version of the staggered grid approach [37], where

$$
\nabla^{2} P \approx \frac{P_{i+2, j}-2 P_{i, j}+P_{i-2, j}}{4 \Delta x^{2}}+\frac{P_{i, j+2}-2 P_{i, j}+P_{i, j-2}}{4 \Delta y^{2}} .
$$


More specifically, instead of using an actual staggered grid, the effect of a staggered grid is modelled through careful discretization of the pressure equation, where the pressure point is located at the center of a $2 \times 2$ block of cells along with velocity points at the faces of this block. This configuration helps with the implementation of a multigrid algorithm as well as takes advantage of the methodology developed in [37]. This approach conserves both mass and energy at the order of local truncation error [16]. Boundary conditions for the pressure need extra ghost points.

\subsection{Lagrangian modelling theory}

\subsubsection{A brief overview of the classical Lagrangian approach}

Lagrangian methods are already well-known, and a detailed review is outside the scope of this article. Briefly, Koumoutsakos [23] reviewed multiscale flow simulation techniques using Lagrangian particle methods. Among various approaches, vortex methods (VM) and smoothed particle hydrodynamics (SPH) methods are efficient, stable, and accurate techniques for computing sharp interfaces such as vortex sheets. Lagrangian methods also have drawbacks, such as significant increases in computational complexity due to periodical re-meshing of distorted Lagrangian mesh. In some applications, these drawbacks can be overcome with an appropriate implementation [38]. The main idea of Lagrangian methods is to avoid a direct discretization of advective derivatives, where the time evolution of inertial effects are modelled by following material elements of a fluid. In this article, we have studied how such an approach helps in accurately modelling mass transfer phenomena. The following Lagrangian method is based on the computation of streamlines, and differs from existing Lagrangian methods [23]. 


\subsubsection{Streamline based Lagrangian modelling}

Let us start with a known divergence free velocity field $v=[u, v]^{T}$, which may have been available through a numerical calculation. Streamlines of this flow are curves, where the velocity field is tangential, and are defined by

$$
\frac{d s_{1}}{u}=\frac{d s_{2}}{v} .
$$

These streamlines, $s=\left[s_{1}, s_{2}\right]^{T}$, can be parameterized, $s(\xi)$, and traced by solving the differential equation

$$
\frac{d s}{d \xi}=v
$$

using an appropriate numerical ODE solver routine [e.g. 39]. When $v$ is the velocity field of an incompressible flow, streamlines will not intersect, begin, or end inside the fluid. For a given physical location with the index $\boldsymbol{k}=\left(k_{1}, k_{2}\right)$, only one streamline, $\boldsymbol{s}_{\boldsymbol{k}}(\xi)$, will pass through this position. For $\xi=0$, if we know a reference position, $\boldsymbol{s}_{\boldsymbol{k}}(0)$, of a streamline that is marked with the index $\boldsymbol{k}$, Eq. (7) can be solved numerically using the initial condition $s_{k}(0)$ to find $s_{k}(\xi)$ for some $\xi>0$ or $\xi<0$. The selection of $\boldsymbol{s}_{\boldsymbol{k}}(0)$ may be arbitrary or $\boldsymbol{k}$ may represent the index of a grid point. For a fixed value of $\xi$, the streamline, $s_{k}(\xi)$, traces physical points $(x, y)$, and hence, we can express the velocity, $\boldsymbol{v}(x, y)$ by $\boldsymbol{v}\left(\boldsymbol{s}_{\boldsymbol{k}}(\xi)\right)$. During the numerical computation of $s_{k}(\xi)$ at discrete values of $\xi$, the right hand side, $v\left(s_{k}\right)$, of Eq. (7) can be updated sequentially since $\boldsymbol{v}(x, y)$ is assumed to be pre-computed. This process traces the parametric curve $s_{k}(\xi)$ that passes through $s_{k}(0)$. To test the process, consider a given velocity, $v=[y,-x]^{T}$, for a solid body rotation flow. Streamlines passing through $s_{k}(0)$ have been computed with this process, and are presented in Fig. 1, where $\circ$ represent the position $s_{k}(0)$.

Since the velocity field is tangential to the streamlines, the velocity is given by 
the directional derivative of a streamline, $s$, along the direction of $s$ such that

$$
v=v \cdot \nabla s
$$

Combining Eq. (7) and Eq. (8), the directional derivative along a streamline can also be parameterized so that

$$
\frac{d}{d \xi}=v \cdot \nabla
$$

and the directional derivative of any scalar field $C(\boldsymbol{x}, t)$ can be written as

$$
\frac{\partial C}{\partial \xi}=v \cdot \nabla C
$$

The directional derivative along a streamline is beneficial to the computation of the numerical solution of (5) when using a fractional time stepping method. The first fractional time step assumes that the total velocity $\boldsymbol{v}=\boldsymbol{u}-\eta \boldsymbol{\nabla} \varphi$ is divergence free, $\boldsymbol{\nabla} \cdot \boldsymbol{v}=0$, and $D=0$ such that (5) reduces to

$$
\frac{\partial C}{\partial t}+\frac{\partial C}{\partial \xi}=0
$$

In Eq. (7), $\xi$ is a time like parameter, and for each value of $\xi$, we have a corresponding streamline position $s(\xi)$. However, in Eq. (10), $\xi$ is a space like parameter, describing the advection of $C$ along a streamline $s(\xi)$. Furthermore, Eq. (10) states that a given concentration field $C^{n}(s(\xi))$ at $t^{n}$ propagates along the streamline $s(\xi)$ at a constant speed, and the solution of (10) can be obtained analytically such that $C(s(\xi), t)=C^{n}(s(\xi-t))$. In the $\xi$-t plane, the characteristic curves of (10) never intersects each other, and hence, the solution of (10) is unique. Therefore, at the first fractional time step, the present method computes $C(\boldsymbol{x}, t)$ according to

$$
C^{n+\frac{1}{2}}(s(\xi), \Delta t)=C^{n}(s(\xi-\Delta t))
$$


where $s(\xi)$ is the streamline position at present time, $t=\Delta t$, and $s(\xi-\Delta t)$ is the streamline position at a previous time, $t=0$.

The second fractional time step accounts for the effect of diffusion, using $C^{n+\frac{1}{2}}(\boldsymbol{s}(\xi), \Delta t)$ as the initial condition assigned at all values of $\boldsymbol{x}=\boldsymbol{s}(\xi)$, where

$$
\frac{\partial C}{\partial t}=\frac{1}{P e} \nabla^{2} C
$$

The diffusion problem (11) is solved with a second order Crank-Nicolson scheme, where a fast multigrid solver is used to solve a linear system of algebraic equations at each time step.

\subsection{Streamline tracing algorithm and computation of $C$}

In the present implementation, we use a uniformly refined structured finite difference mesh of the domain $\left[x_{\min }, x_{\max }\right] \times\left[y_{\min }, y_{\max }\right]$, where each grid point $\left(x_{i}, y_{j}\right)$ is given by $x_{i}=x_{\min }+i \Delta x$ and $y_{j}=y_{\min }+j \Delta y$, using $\Delta x$ and $\Delta y$ as step sizes in the $x$ and $y$ directions respectively. There are $n_{x} \times n_{y}$ cells, and the position

$\left(x_{i}, y_{j}\right)$ is at the center of a cell. The notation $C_{i j}^{n}$ represents $C(\boldsymbol{x}, t)$ at cell $(i, j)$ for time $t^{n}$ or $C\left(s_{k}(0), t^{n}\right)$ at the streamline position $s_{k}(0)$ that is on the cell center $\left(x_{i}, y_{j}\right)$. Suppose that we want to compute $C_{i j}^{n+1}$ by tracing a streamline.

As described above, a streamline that originates from a cell, $\boldsymbol{s}_{\boldsymbol{k}}(0)=\left(x_{i}, y_{j}\right)$, can be traced forward from Eq. (7) for $\xi \in[0, \Delta t]$. Using a prescribed solid body rotation flow $\boldsymbol{v}=[y,-x]^{T}$, we have verified that a finite number of computed streamlines do not intersect each other (plots are not shown). In Fig. (1), the position $s_{k}(0)$ is denoted by $\circ$, and all streamlines, $s_{k}(\xi)$, passing through $\circ$, for one time step have been presented. Clearly, $\boldsymbol{s}_{\boldsymbol{k}}(0)$ is a grid point, but $\boldsymbol{s}_{\boldsymbol{k}}(\xi)$ is not. We can neither store $C\left(s_{k}(\xi)\right)$ nor use $\boldsymbol{v}\left(s_{k}(\xi)\right)$ because our dependent variables are stored on grid points only. As described above, the proposed method computes 
$C^{n+1 / 2}\left(s_{k}(\xi)\right)=C^{n}\left(s_{k}(0)\right)$ from (10) at the first fractional step, which does not provide with $C_{i j}^{n+1 / 2}$ because $s_{k}(\xi)$ is not on a grid point. Clearly, $C_{i j}^{n+1 / 2}$ is an unknown quantity (on a o position), which can be interpolated from known quantities $C^{n+1 / 2}\left(s_{k}(\xi)\right)$ at $s_{k}(\xi)$ positions those are neighbors of a o position. Looking at any position $\circ$ in Fig. 1, one finds immediately that a standard interpolation method is not applicable for interpolating $C_{i j}^{n+1 / 2}$ from values of $C^{n+1 / 2}\left(s_{k}(\xi)\right)$ those are at surrounding streamline positions. Using the principle of mass conservation (10), we have proposed the following parcel advection methodology for updating $C_{i j}^{n+1 / 2}$ at each grid cell $(i, j)$.

Let us start with the assumption that fluid occupies the entire domain, and that a parcel is the fluid that is contained in one cell. There are $n_{x} \times n_{y}$ parcels of the same size, and each parcel is tagged with an individual concentration value, $C_{i j}^{n}$. In other words, $C_{i j}^{n}$ represents the mean concentration value of the parcel of fluid that occupies the corresponding cell. According to the streamline notation, $C_{i j}^{n}=C\left(s_{k}(0)\right)$. Thus, $C\left(s_{k}(\xi)\right)$ represents the mean concentration of a fluid parcel at the streamline position $s_{k}(\xi)$. Eq. (10) confirms that $C\left(s_{k}(0)\right)$ will be redistributed to $s_{k}(\xi)$. As soon as the streamline has been traced, we can distribute the concentration $C\left(s_{k}(0)\right)$ to another parcel of same size located at $\boldsymbol{s}_{\boldsymbol{k}}(\xi)$. With this re-distribution of $C$, the total amount of $C$ will never change. The idea has been depicted schematically in Fig. 1 with broken lines, showing that the concentration of the parcel at $\circ$ may be distributed at $\bullet$ according to a portion of the overlapping area. Clearly, a parcel of the same size at $\bullet$ may overlap at most 4 cells. The idea is to distribute $C\left(s_{k}(\xi)\right)$ into overlapping cells.

Let $s_{k}(0)$ be at the center ' $\circ$ ' of the cell $(i, j)$ and $s_{k}(\xi)$ be within the cell $(i-1, j+1)$, but not at the center. Since the total concentration of the parcel 
at $\circ$ is $A C_{i j}^{n}$, this parcel will contribute to changing the value of $C$ in cells $(i, j)$, $(i+1, j),(i, j+1)$, and $(i+1, j+1)$ according to $A_{1} C_{i j}^{n}, A_{2} C_{i j}^{n}, A_{3} C_{i j}^{n}, A_{4} C_{i j}^{n}$, where $A_{i}$ 's are portions of corresponding overlapping areas as shown in Fig. 1. Each streamline will contribute $C_{i j}^{n}$ to at most 4 cells, covering all of the initial concentration according to the computed overlapped area.

During the next time step, it is natural to trace the streamline that passes through $s(\xi)$ using the velocity at $s(\xi)$. Since $s(\xi)$ is not a grid point, $v(s(\xi))$ is not known, and may be computed from the neighboring cells according to

$$
\boldsymbol{v}(\boldsymbol{s}(\xi))=\frac{1}{A}\left(A_{1} \boldsymbol{v}_{i, j}+A_{2} \boldsymbol{v}_{i+1, j}+A_{3} \boldsymbol{v}_{i, j+1}+A_{4} \boldsymbol{v}_{i+1, j+1}\right),
$$

where $A$ is the area (volume) of the $2 \mathrm{D}$ parcel, and $A_{1}, A_{2}, A_{3}, A_{4}$ are fractional areas as depicted in Fig.1.

This procedure traces all streamlines emerging initially from the center of each computational cell. At each step, the velocity for computing each streamline is updated, assuming the velocity field has already been computed. The concentration for each computational cell is updated at each time step as soon as the streamline has been traced.

\section{Comparison with a reference model and the heuristic mass transfer theory}

This section presents the benefits of the Lagrangian model with respect to a commonly used equivalent reference Eulerian model. A heuristic mass transfer methodology is studied to explain the theory of numerical mass diffusion and dispersion so that the source of error as time elapses can be identified theoretically [31]. Although not in common practice, the heurisitic theory presented by Warming and Hyett [31] is useful to understand the source of error that destroys the quality of an Eulerian model, and is also useful to explore the benefits 
of the Lagrangian modelling approach. Further details of the heuristic method can also be found in [40].

\subsection{Two reference finite difference schemes}

Let us consider a finite difference discretization of a one-dimensional mass transfer problem

$$
\frac{\partial C}{\partial t}+v \frac{\partial C}{\partial x}=0
$$

as written below

$$
\frac{C_{i}^{n+1}-C_{i}^{n}}{\Delta t}+v \frac{C_{i+1}^{n}-C_{i-1}^{n}}{2 \Delta x}=0
$$

where $C_{i}^{n}=C(i \Delta x, n \Delta t)$ is an evaluation of the continuous concentration field $C(x, t)$ on the $i$-th grid point at $n$-th time step. This is an explicit method with a truncation error $O\left(\Delta x^{2}, \Delta t\right)$. In the limit of $\Delta x \rightarrow 0, \Delta t \rightarrow 0$, the truncation error vanishes, and the finite difference Eq. (13) approaches the actual partial differential equation (PDE) (12). Since the scheme (13) is unconditionally unstable, it is a common practice to use the upwind scheme:

$$
\frac{C_{i}^{n+1}-C_{i}^{n}}{\Delta t}+v \frac{C_{i}^{n}-C_{i-1}^{n}}{\Delta x}=0 \text { for } v>0,
$$

which has a truncation error $O(\Delta x, \Delta t)$. The scheme (14) is conditionally stable if $\left|\frac{v \Delta t}{\Delta x}\right| \leq 1$, which means that if $\Delta t$ is restricted, the numerical error does not grow spontaneously. However, this fundamental concept of numerical stability does not confirm whether the scheme is still appropriate for the multiphysics problem of electro-osmotic mass transport or a higher order version of (14) is needed. Neither the order of accuracy nor the stability condition tells us how this scheme would predict a flow as time elapses; one can only see the effect of error accumulation after computation of the flow for many time steps. Hereinafter, we will refer to (14) as the reference Eulerian model. 
The following heuristic theory explains the behavior of a scheme when numerical integration time elapses. To the best of authors' knowledge, this methodology is not widely used by the heat and mass transfer research community. Although, the following presentation has used a usual upwind scheme, the analysis is applicable to other higher order upwind schemes.

\subsection{Modified partial differential equation}

We can expand each of the function evaluations $C_{i}^{n+1}, C_{i+1}^{n}$, and $C_{i-1}^{n}$ about the point $(i \Delta x, n \Delta t)$ using a Taylor series, which converts the finite difference Eq. (13) into the continuous form

$$
\frac{\partial C}{\partial t}+v \frac{\partial C}{\partial x}=-\frac{\Delta t v^{2}}{2} \frac{\partial^{2} C}{\partial x^{2}}+O\left(\Delta x^{2}, \Delta t^{2}\right)
$$

Eq. (15) is called the modified partial differential equation (MPDE), and MPDEs for similar schemes can be obtained using table I from Warming and Hyett [31]. This is the actual equation solved by scheme (13) instead of Eq. (12). Neglecting terms of order $O\left(\Delta x^{2}, \Delta t^{2}\right)$ and higher, we see that the MPDE contains an extra term with an effective negative mass diffusion, $v_{\text {art }}=-v^{2} \Delta t / 2$, and hence, Eq. (15) produces an unstable solution. Similarly, the MPDE for (14) takes the form

$$
\frac{\partial C}{\partial t}+v \frac{\partial C}{\partial x}=\frac{v \Delta x}{2}\left(1-\frac{v \Delta t}{\Delta x}\right) \frac{\partial^{2} C}{\partial x^{2}}+O\left(\Delta x^{2}, \Delta t^{2}, \Delta x \Delta t\right) .
$$

Neglecting $O\left(\Delta x^{2}, \Delta t^{2}\right)$ terms, the leading order error in the MPDE (16) will introduce an effective positive mass diffusion if $v \Delta t / \Delta x \leq 1$, and the solution of (16) will diffuse in time. Hence, the numerical solution will diverge from the actual solution after many time steps. Clearly, the artificial mass diffusion $v_{\text {art }}=v \Delta x / 2(1-v \Delta t / \Delta x)$ would infer the rate of divergence, and one must have 
extremely small values for both $\Delta x$ and $\Delta t$ in order to have $v_{\text {art }} \ll 1$. This analysis can be generalized to study the effects of artificial mass diffusion if the mass transport problem (12) is directly discretized (with $\mathrm{FD} / \mathrm{FV} / \mathrm{FE}$ ) on an Eulerian mesh.

\subsection{The heuristic analysis for computational mass transfer}

It may be more appropriate to recast briefly - the heuristic method of Warming and Hyett [31] to the computational mass transfer problem - without going to the details of derivations, using the general form of the mass transport equation

$$
\frac{\partial C}{\partial t}+\mathcal{L}(C)=0
$$

where $\mathcal{L}(C)$ represents the spatial derivative terms. If all spatial derivatives of Eq. (17) are discretized with a collocation based FD/FV/FE method, the corresponding MPDE for (17) may be written in the following general form

$$
\frac{\partial C}{\partial t}+\mathcal{L}(C)=\sum_{p=1}^{\infty} \lambda_{2 p} \frac{\partial^{2 p} C}{\partial x^{2 p}}+\sum_{p=0}^{\infty} \lambda_{2 p+1} \frac{\partial^{2 p+1} C}{\partial x^{2 p+1}}
$$

where $\lambda_{2 p}$ or $\lambda_{2 p+1}$ is a function of $\Delta x^{p}$ and/or $\Delta t^{p}$. On the right hand side of (18), the even order derivative terms will introduce artificial diffusion of mass, and the odd order derivative terms will introduce artificial dispersion of mass. As shown by Warming and Hyett [31], a necessary condition for the stability of a finite difference scheme is

$$
(-1)^{p_{c}-1} \lambda_{2 p_{c}}>0
$$

where $p_{c}$ corresponds to the lowest order non-zero term in the right hand side of (18). For the finite difference scheme (13), we have $p_{c}=1$ and $(-1)^{p_{c}-1} \lambda_{2 p_{c}}=$ 
$-\Delta t v^{2} / 2$, and hence, the scheme does not satisfy the necessary condition for stability. Similarly, we can show that the scheme (14) does satisfy the stability condition (19). One observes that a discretization must be adopted so that the MPDE does not contain any even order derivatives. Otherwise, small values of $\Delta x$ and $\Delta t$ are necessary to make $\lambda_{2 p_{c}}$ small. This study encourages us to develop a methodology where the numerical scheme yields an MPDE with no even order derivative terms to at least the lowest order error term.

\subsection{Spurious mass diffusion of the reference model}

MPDE (16) asserts that the reference model (14) introduces a leading order error that can be quantified by a mesh Peclet number such that $\frac{1}{P e}=\frac{v \Delta x}{2}\left(1-\frac{v \Delta t}{\Delta x}\right)$. We keep the same symbol $P e$ (for 'Peclet' and 'mesh Peclet' number) because mesh Peclet number is used only in the present subsection. Clearly, the error may be minimized by reducing $\Delta x$ and $\Delta t$, and a large mesh $P e$ indicates a small error. To study the effect of artificial mass diffusion due to this error in the reference model using the heuristic theory, consider a simplified model of (16), given by

$$
\frac{\partial C}{\partial t}=\frac{1}{P e} \frac{\partial^{2} C}{\partial x^{2}}
$$

along with the initial Gaussian distribution

$$
C(x, 0)=\frac{\exp \left(\frac{-x^{2}}{4 / P e}\right)}{\sqrt{4 \pi / P e}},
$$

where $C$ decays rapidly with respect to $x$. Eq. (20) models the effects of artificial mass diffusion as time elapses, and can be solved along with the boundary condition

$$
C \rightarrow 0 \quad \text { as } \quad|x| \rightarrow \infty
$$


such that

$$
C(x, t)=\frac{\exp \left(\frac{-x^{2}}{4 / P e(t+1)}\right)}{\sqrt{4 \pi / P e(t+1)}} .
$$

Using the scales (e.g. $h, U$ ) listed in Table 2, we can estimate a dimensional diffusion coefficient $D$ for a corresponding value of the mesh Peclet number $P e$. The solution (22) has been plotted in Fig. 2 for several values of $\frac{1}{P e}, 2 \times 10^{-3}(D=$ $\left.10^{-10} \mathrm{~m}^{2} / \mathrm{s}\right), 10^{-3}\left(D=5 \times 10^{-11} \mathrm{~m}^{2} / \mathrm{s}\right), 5 \times 10^{-4}\left(D=2.5 \times 10^{-11} \mathrm{~m}^{2} / \mathrm{s}\right), 10^{-4}(D=$ $\left.5 \times 10^{-12} \mathrm{~m}^{2} / \mathrm{s}\right), 5 \times 10^{-5}\left(D=2.5 \times 10^{-12} \mathrm{~m}^{2} / \mathrm{s}\right)$. Clearly, only a negligible physical diffusion $\left(10^{-10} \mathrm{~m}^{2} / \mathrm{s} \leq D \leq 2.5 \times 10^{-12} \mathrm{~m}^{2} / \mathrm{s}\right)$ is modeled with $2 \times 10^{-3} \leq$ $\frac{1}{P e} \leq 5 \times 10^{-5}$. Since the estimated value of $D$ is small, the interpretation of the leading order error in (16) may be misleading. With equivalent scaling, Fig. 2 exhibits notable spreading of $C$ for only about a factor of 32 decrease in the mesh Peclet number $P e$ due to the error. This means the error identified by (16) may have a serious impact in some cases. As explained below, one can estimate the computational burden needed to avoid the artificial mass diffusion.

In a situation where the physical diffusion coefficient is $D=2.5 \times 10^{-12} \mathrm{~m}^{2} / \mathrm{s}$, the actual Peclet number takes a value $1 / P e=5 \times 10^{-5}$. In this case, the numerical solution should agree with the narrow profile '_-' in Fig. 2. However, if the mesh Peclet number for the MPDE differs from actual $P e$ such that $\frac{1}{P e}=2 \times 10^{-3}$ due to a coarse resolution ( $\Delta x$ and $\Delta t$ ), the numerical solution may converge to the shallow profile '-০-০-' in Fig. 2 instead of converging to the expected narrow profile '-', which is a clear divergence of the solution. In the present example, the artificial mass diffusion can be minimized from $2 \times 10^{-3}$ to $5 \times 10^{-5}$ by refining the mesh uniformly at least a factor of 32 in each direction, as well as the time step $\Delta t$, in order to get a mesh Peclet number such that $\frac{1}{P e}=5 \times 10^{-5}$. To do so with the reference model, the computational complexity would increase by a factor of 
32,768 (about $10^{4}$ ) for a 2D simulation, and by a factor of $1,048,576$ (about $10^{6}$ ) for a $3 \mathrm{D}$ simulation, assuming that both $\Delta x$ and $\Delta t$ must be reduced. Note that such an increase of grid points may have other numerical artifacts, and clearly, the approach is not appropriate.

The theoretical study presented in this section shows that a careful investigation of numerical error in a classical computational model is necessary, where the transport term is discretized directly on an Eulerian mesh. The proposed Lagrangian model is one way to eliminate the spurious mass diffusion, and brings computational advantage saving the complexity by about a factor of $10^{4}$ for the

2 D electro-osmotic mass transfer problem $\left(2 \times 10^{-3} \leq \frac{1}{P e} \leq 5 \times 10^{-5}\right)$. Improving the solution of a computational model without overburdening the computational work is the second of two unresolved challenges discussed in the introduction. The proposed development is now validated with numerical experiments. Using 2D simulations of both the Lagrangian model and the reference model, we have verified in the next section that the Lagrangian model has addressed the modelling problem that has been reported in the present section.

\section{Results, verification, and validation}

In all color figures presented in this section, blue and red colors represent the dimensionless minimum and maximum values respectively of the corresponding quantity, and the yellow color indicate a zero. Further, $\mathrm{x}$-axis represents $x / h$ and $y$ axis represents $y / h$, unless otherwise indicated in the corresponding figure, where $h$ is the length scale. 


\subsection{Incompressible flow computation}

The 2D Taylor-Green vortex flow is a model that is widely used to verify numerical accuracy of incompressible flow computation, where the velocity and pressure satisfying the incompressible Navier-Stokes equation are defined by

$$
\begin{aligned}
u(x, y, t) & =-\cos (2 \pi x) \sin (2 \pi y) \exp \left(-8 \pi^{2} t / R e\right) \\
v(x, y, t) & =\sin (2 \pi x) \cos (2 \pi y) \exp \left(-8 \pi^{2} t / R e\right) \\
P(x, y, t) & =-\frac{1}{4}(\cos (4 \pi x)+\cos (4 \pi y)) \exp \left(-16 \pi^{2} t / R e\right) .
\end{aligned}
$$

Fig. 3 shows a good agreement between the simulated velocity and the exact velocity (23). This model has produced equivalent results with various values of $\Delta x$, $\Delta y$, and $\Delta t$. These tests confirm the accuracy of the velocity solver.

\subsection{Numerical Simulation of Electro-Osmotic Flow}

To simulate an electro-osmotic flow, necessary dimensional parameters and scales are listed in Table 1 and Table 2 respectively. The initial concentration field, $C(x, 0)$, having a non-zero value identified with the red color is shown in Fig. 4(o). The values of dimensionless parameters for the present set of simulations are

$\frac{1}{R e}=20, \quad 5 \times 10^{-5} \leq \frac{1}{P e} \leq 2 \times 10^{-3}, \quad \alpha=1.27 \times 10^{5}, \quad \kappa^{2}=4280$, and $\eta=0.28$, where an increase in $P e$ reflects a decrease in the dimensional diffusion coefficient $D$. For $\frac{1}{P e}=5 \times 10^{-5}$, we have $D=2.5 \times 10^{-12} \mathrm{~m}^{2} / \mathrm{s}$. With such a small value of $D$, the effect of diffusion is negligible. Therefore, an initial scalar plug $C(\boldsymbol{x}, 0)$ that is transported in a microchannel with an electro-osmotic flow is not expected to spread or diffuse significantly, i.e. mass transport is dominant over mass diffusion. Furthermore, Eq. (5) also states that $\int_{V} C^{2}(\boldsymbol{x}, t) d V$ is conserved for an arbitrary 
control volume $V$ with no solid boundaries if $D=0$. In applications, $D \neq 0$, and a flow is often confined within solid boundaries. Hence, an understanding of the transport of $C$ with respect to increasing/decreasing $P e$ explains how accurately the numerical model has simulated the actual mass transfer problem.

To test if the present model simulates the flow in a microchannel so that the mass transfer remains dominant over mass diffusion, a number of numerical experiments have been performed using both the proposed Lagrangian model and a reference Eulerian model. Some representative and equivalent sets of datum from both model have been presented in Fig. 4. Clearly, the Lagrangian model resolves the effect of increasing $P e$ more accurately compared to the Eulerian model because the spatial spreading of the initial $C$ (e.g. Fig. 4(o)) decreases with respect to increasing $P e$.

\subsection{A quantitative assessment and theoretical justification for the proposed model}

For the first set of numerical experiments, we have presented in Fig. 4(a-e) the time evolution of the initial $C$ using the Lagrangian model for $\frac{1}{P e}=2 \times 10^{-3}, 10^{-3}$, $5 \times 10^{-4}, 10^{-4}$, and $5 \times 10^{-5}$ at $t=4.4$, when the sample has been transported to about the middle of the channel. Note that the diffusion is strong for $\frac{1}{P e}=2 \times 10^{-3}$,

but weak for $\frac{1}{P e}=5 \times 10^{-5}$. As expected, the increase of $P e$ has reduced the diffusion of the concentration field $C$.

In the second set of experiments, we have repeated these simulations using the Eulerian model, and the results are presented in Fig. $4(f-j)$, where the effect of increasing $\mathrm{Pe}$ is clearly not resolved. The Eulerian model has failed to simulate the transport dominated flow.

First, the failure of the Eulerian model is explained from the theory presented by (16), where $v_{\text {art }}$ is constant for fixed $\Delta x$ and $\Delta t$. Although $P e$ has been in- 
creased, artificial mass diffusion remains dominant over mass transfer phenomena. Second, the Lagrangian simulation of mass transfer is fully consistent with theoretical analysis presented in section 3.3 and in Warming and Hyett [31]. Care must be taken while interpreting these Lagrangian simulations because the heuristic theory, when applied to an equivalent Eulerian model, only indicate that artificial diffusion is not dominant over physical diffusion in the present Lagrangian simulation. Under similar computational and physical circumstances, the Lagrangian model resolves the physics, when the diffusion coefficient varies between $O\left(10^{-10} \mathrm{~m}^{2} / \mathrm{s}\right)$ and $O\left(10^{-12} \mathrm{~m}^{2} / \mathrm{s}\right)$. The ability of resolving variation in such a small diffusion coefficient is one important aspect of the present Lagrangian model.

From the distributions of $C$ presented in Figs 4(a-e), we have computed the profiles of $C$ along the line $y=0.5$, and have presented the result in Fig. 5(a). For the ease of comparison, the initial profile $C(x, 0.5,0)$ from data in Fig. 4(o) has been translated and presented in Fig. 5(a). Clearly, the width of the Gaussian mass distribution increases if $P e$ decreases, which is in good agreement with theoretical model of mass diffusion. In addition, the height of the Gaussian profile is proportional to $\sqrt{\frac{1}{P e}}(e . g$. , Eq. (21)). The maximum values for each distribution of $C$ from Fig. 4( $a-j)$ have been compared with the theoretical height $\sqrt{\frac{1}{P e}}$ in Fig. 5(b). The Lagrangian model appears to fit well with the theoretical result, which shows a large deviation from Eulerian model. These results present a quantitative assessment for the proposed model's ability to resolve mass transfer phenomena, and bring potential improvements to the field of computational mass transfer modelling. 


\subsection{Quality of solution as time elapses}

Let us now study the performance of the Lagrangian model at different time intervals, where streamlines are computed by solving the system of ODEs (7) numerically, using a forward in time method. It is important to understand if truncation errors accumulate, the global solution will be affected as integration time elapses. This is examined by simulating electro-osmotic transport at three different time intervals, and by comparing simulations using the Lagrangian and the Eulerian models. In Figs 6 (a)-(c), simulated $C(x, y, t)$ profiles using the Lagrangian model at $t=2.2, t=4.4$, and $t=6.6$ have been presented, where we see that the concentration field has roughly the same width as time increases. Since $\frac{1}{P e}=5 \times 10^{-5}$, the diffusion is negligible, and we do not expect to see widening of the initial sample as it is transported along the channel. The Lagrangian model's result does not indicate any accumulated diffusive behavior at these three time intervals. However, plots of $C(x, y, t)$ in Figs 6 (d)-(f) exhibit a noticeable widening in the concentration profile with increasing time. These plots have been produced using the Eulerian model, where the accumulation of error as time increases is clearly visible. Note that both the Lagrangian and Eulerian models have equivalent order of accuracy. These results indicate that the Lagrangian model has controlled the error at various time intervals as soon as the sample is transported along the channel, which is not the case with the Eulerian model. This means that the numerical treatment of advection along the streamline helps to avoid the error accumulation compared to a direct discretization of advection terms.

\subsection{Comparison between electro-osmotic and hydrodynamic transport}

Patankar and $\mathrm{Hu}[8]$ explained that an electro-osmotic pumping is optimized when a rectangular sample plug is used, i.e., the distribution of $C(\boldsymbol{x}, t)$ needs 
to be approximately rectangular as in Fig. 7(a). Pressure gradient forces may lead to a parabolic distribution of the transported sample in an experiment. The present model can be used to study the optimal pressure gradient that needs to be maintained during an experiment. Here, we have studied the development of the parabolic shape when the sample is transported with combined electroosmotic and hydrodynamic forcing. Fig. 7 displays the concentration fields at $t=4.4$ with varying values of the applied pressure $P_{0}$ at the input. As $P_{0}$ is increased, the parabolic nature of the concentration field, which is characteristic of a pressure-driven Poiseuille flow, becomes more dominant. This experiment confirms that the proposed Lagrangian model simulates both the hydrodynamic and the electro-osmotic transport without introducing artificial damping. Moreover, the Lagrangian model has the potential to analyze the flow and to determine the order of magnitude of the pressure drop a priori to a typical experiment if other equivalent parameters are known.

\section{Conclusion, contribution, and future direction}

In this research, we have developed a Lagrangian methodology for modelling electro-kinetic mass transport in microchannels, where typical length scales of the flow are on the order of microns. Our methodology proposes to model the time evolution of mass transport phenomena using a streamline coordinate, where streamlines are computed numerically from a given velocity field. In this approach, a two- or three-dimensional advection equation is converted into a onedimensional equation in the streamline coordinates, which is solve analytically. Since streamlines are attached to the dynamics of a flow, this helps to resolve the flow more accurately as well as saves computational time. Using a fractional time 
stepping algorithm, the effect of diffusion is modelled with a multilevel method employing a second order trapezoidal or Crank-Nicolson scheme. When the mesh is refined for resolving the Debye layer and other interfacial structures, the multilevel solver helps to preserve a linear relatioship between the number of grid points and the computing time. To transfer data between streamlines and grid points, an optimal interpolation scheme is studied without violating the desired accuracy. We have studied how the heuristic theory of Warming and Hyett [31] can potentially be used to identify source of errors destroying the physical characteristics of a simulated flow. Comparisons with a computationally equivalent Eulerian model as well as with theoretical estimates have revealed that the Lagrangian model simulates the flow more efficiently.

There are several potential extensions of this work to be considered. First, the approach can be extended to simulate a 3D electro-kinetic mass transfer problem in a complex geometry. For this purpose, the present development has been implemented in the primitive variables - velocity and pressure. The present multigrid routine must be extended and verified for a 3D flow. Second, the model can be extended to take advantage of multi-core clusters, implementing and extending the present code with Message Passing Interface (MPI) libraries. Third, Figs 4 and 7 indicate that the spatial mesh may only be refined locally in the region, where $C$ has a sharp gradient. Thus, a suitable adaptive mesh refinement (AMR) approach may improve the present Lagrangian model. To the best of our knowledge, a combined application of Lagrangian approach and AMR has not been studied extensively despite a few potential attempts. Such an extension of this work requires the development of advanced data structures, such as a binary tree, and the implementation of polynomials with compact support so that discretiza- 
tion of governing PDEs can be implemented in such a way that takes advantage of the ease of finite difference techniques. These extensions are currently in progress.

\section{Acknowledgements}

JMA acknowledges financial support from the National Science and Research Council (NSERC), Canada.

\section{References}

[1] H. S. Ahn, C. Lee, J. Kim, M. H. Kim, The effect of capillary wicking action of micro/nano structures on pool boiling critical heat flux, International Journal of Heat and Mass Transfer 55 (2012) 89 - 92.

[2] M. Akhavan-Behabadi, M. F. Pakdaman, M. Ghazvini, Experimental investigation on the convective heat transfer of nanofluid flow inside vertical helically coiled tubes under uniform wall temperature condition, International Communications in Heat and Mass Transfer (2012). In press.

[3] R. A. Hart, A. K. da Silva, Experimental thermal-hydraulic evaluation of constructal microfluidic structures under fully constrained conditions, International Journal of Heat and Mass Transfer 54 (2011) 3661 -71.

[4] D. Kawashima, Y. Asako, First law analysis for viscous dissipation in liquid flows in micro-channels, International Journal of Heat and Mass Transfer 55 (2012) $2244-8$.

[5] M. J. Canny, Flow and transport in plants, Annual Review of Fluid Mechanics 9 (1977) 275-96. 
[6] Y. Sui, C. Teo, P. Lee, Direct numerical simulation of fluid flow and heat transfer in periodic wavy channels with rectangular cross-sections, International Journal of Heat and Mass Transfer 55 (2012) 73 - 88.

[7] A. Lee, V. Timchenko, G. Yeoh, J. Reizes, Three-dimensional modelling of fluid flow and heat transfer in micro-channels with synthetic jet, International Journal of Heat and Mass Transfer 55 (2012) 198 - 213.

[8] N. A. Patankar, H. H. Hu, Numerical simulation of electro-osmotic flow, Anal. Chem 70 (1998) 1870-81.

[9] D. J. Harrison, A. Manz, Z. Fan, H. Lüdi, H. M. Widmer, Capillary electrophoresis and sample injection systems integrated on a planar glass chip, Anal. Chem. 64 (1992) 1926-32.

[10] F. Bianchi, R. Ferrigno, H. H. Girault, Finite element simulation of an electroosmotic-driven flow division at a T-junction of microscale dimensions, Analytical Chemistry 72 (2000) 1987-93.

[11] C.-K. Chen, C.-C. Cho, Electrokinetically driven flow mixing utilizing chaotic electric fields, Microfluidics and Nanofluidics 5 (2008) 785-93.

[12] J. G. Santiago, Electroosmotic flows in microchannels with finite inertial and pressure forces, Analytical Chemistry 73 (2001) 2353-65.

[13] Y. Aboelkassem, Numerical simulation of electroosmotic complex flow patterns in a microchannel, Computers \& Fluids 52 (2011) $104-15$.

[14] K. Kim, H. S. Kwak, T.-H. Song, A numerical model for simulating elec- 
troosmotic micro- and nanochannel flows under non-boltzmann equilibrium, Fluid Dynamics Research 43 (2011) 041401-13.

[15] J. M. Alam, A Fully Lagrangian Advection Scheme for Electro-Osmotic Flow, Master's thesis, University of Alberta, Edmonton, AB, 2000.

[16] J. M. Alam, J. C. Bowman, Energy conserving simulation of incompressible electro-osmotic and pressure driven flow, Theoret. Comput. Fluid Dynamics. 16 (2002) 133-50.

[17] S. V. Ermakov, S. C. Jacobson, J. M. Ramsey, Computer simulations of electrokinetic transport in microfabricated channel structures, Anal. Chem. 70 (1998) 4494-504.

[18] A. Chatterjee, Generalized numerical formulations for multi-physics microfluidics-type applications, Journal of Micromechanics and Microengineering 13 (2003) 758-67.

[19] A. Arnold, P. Nithiarasu, P. Tucker, Finite element modelling of electroosmotic flows on unstructured meshes, International Journal of Numerical Methods for Heat \& Fluid Flow 18 (2008) 67-82.

[20] D. P. J. Barz, P. Ehrhard, Fully coupled model for electrokinetic flow and transport in microchannels, Proc. Appl. Math. Mech 5 (2005) 535-6.

[21] C.-K. Chen, C.-C. Cho, A combined active/passive scheme for enhancing the mixing efficiency of microfluidic devices, Chemical Engineering Science 63 (2008) $3081-7$. 
[22] J. A. Sethian, P. Smereka, Level set methods for fluid interfaces, Annual Review of Fluid Mechanics 35 (2003) 341-72.

[23] P. Koumoutsakos, Multiscale flow simulations using particles, Annual Review of Fluid Mechanics 37 (2005) 457-87.

[24] A. Harten, B. Engquist, S. Osher, S. R. Chakravarthy, Uniformly high order accurate essentially non-oscillatory schemes, iii, Journal of Computational Physics 131 (1997) $3-47$.

[25] Y. Gao, T. Wong, J. Chai, C. Yang, K. Ooi, Numerical simulation of twofluid electroosmotic flow in microchannels, International Journal of Heat and Mass Transfer 48 (2005) $5103-11$.

[26] J. M. Alam, N. K.-R. Kevlahan, O. V. Vasilyev, Z. Hossain, A multiresolution model for the simulation of transient heat and mass transfer, $\mathrm{Nu}-$ merical Heat Transfer, Part B 61 (2012) 1-24.

[27] H. Park, Y. Choi, Electroosmotic flow driven by oscillating zeta potentials: Comparison of the Poisson-Boltzmann model, the Debye-Hückel model and the Nernst-Planck model, International Journal of Heat and Mass Transfer 52 (2009) $4279-95$.

[28] S. R. Mathur, J. Y. Murthy, A multigrid method for the Poisson-NernstPlanck equations, International Journal of Heat and Mass Transfer 52 (2009) $4031-9$.

[29] W. E, J.-G. Liu, Projection method I: Convergence and numerical boundary layers, SIAM Journal on Numerical Analysis 32 (1995) 1017-57. 
[30] B. Kirby, Micro- And Nanoscale Fluid Mechanics: Transport in Microfluidic Devices, Cambridge University Press, New York, first edition, 2010.

[31] R. F. Warming, B. J. Hyett, The modified equation approach to the stability and accuracy analysis of finite-difference methods, Journal of Computational Physics 14 (1974) 159-79.

[32] G. K. Batchelor, Developments in microhydrodyanmics, in: W. T. Koiter (Ed.), Theoretical and applied mechanics, The 14th IUTAM Congress, 30 August-4 September 1976, North-Holland publishing company, Amsterdam, New-York, Oxford, Delft, Netherlands, 1977, pp. 33-55.

[33] H. Stone, A. Stroock, A. Ajdari, Engineering flows in small devices, Annual Review of Fluid Mechanics 36 (2004) 381-411.

[34] P. Debye, E. Hückel, Zur Theorie der Electrolyte, Phys. Z. 24 (1923) 185305.

[35] H. L. F. Helmholtz, Studies of electric boundary layers, Annals Physik. 7 (1879) 337-82.

[36] D. A. Saville, Electrokinetic effects with small particles, Annual Review of Fluid Mechanics 9 (1977) 321-37.

[37] F. H. Harlow, J. E. Welch, Numerical calculation of time-dependent viscous incompressible flow of fluid with free surface, Physics of Fluids 8 (1965) 2182-9.

[38] J. M. Alam, J. C. Lin, Towards a fully lagrangian atmospheric modelling system, Monthly Weather Review 136 (2008) 4653-67. 
[39] L. Shampine, J. R. Allen, S. Pruess, Fundamentals of numerical computing, John Wiley \& Sons, Inc, first edition, 1997.

[40] J. C. Tannehill, D. A. Anderson, R. H. Pletcher, Comput. Fluid Mech. Heat Trans., Taylor and Francis, 1997. 


\begin{tabular}{lll}
\hline Kinematic viscosity of buffer solution & $v$ & $10^{-6} \mathrm{~m}^{2} / \mathrm{s}$ \\
Electrophoretic mobility & $\tilde{\mu}$ & $1.4 \times 10^{-8} \mathrm{~m}^{2} /(\mathrm{V} \mathrm{s})$ \\
Boltzmann constant & $\mathcal{B}$ & $1.381 \times 10^{-23} \mathrm{~J} / \mathrm{K}$ \\
Elementary charge & $e$ & $1.602 \times 10^{-19} \mathrm{C}$ \\
Temperature & $T$ & $300 \mathrm{~K}$ \\
Permittivity of buffer solution & $\epsilon$ & $6.95 \times 10^{-10} \mathrm{C}^{2} / \mathrm{N} \mathrm{m}^{2}$ \\
Buffer ion density & $n$ & $3.18 \times 10^{-4} \mathrm{~mol}^{3} \mathrm{~m}^{3}$ \\
Avogadro's number & $\mathcal{A}$ & $6.022 \times 10^{23} \mathrm{~mol}^{-1}$ \\
Debye layer thickness & $\mathcal{D}$ & $765 \mathrm{~nm}$ \\
\hline
\end{tabular}

Table 1: Dimensional parameters for modelling electro-kinetic mass transfer in a microchannel. These parameters have been used in Section 4. 


\begin{tabular}{|lll|}
\hline Channel length & $H$ & $4 \times 10^{-4} \mathrm{~m}$ \\
Channel width & $h$ & $5 \times 10^{-5} \mathrm{~m}$ \\
Velocity scale & $U$ & $10^{-3} \mathrm{~m} / \mathrm{s}$ \\
Time scale & $h / U$ & $0.05 \mathrm{~s}$ \\
Pressure scale & $\rho U^{2}$ & $10^{-3} \mathrm{~Pa}$ \\
Applied pressure at the input & $P_{0}$ & $48 \times \rho U^{2}$ \\
Pressure at the output & $P_{1}$ & $1 \times \rho U^{2}$ \\
Wall potential scale & $\zeta$ & $107 \mathrm{mV}$ \\
External potential scale & $\phi$ & $1 \mathrm{~V}$ \\
Applied voltage at the input & $\Phi$ & $4 \times \phi$ \\
Applied voltage at the output & & $0 \mathrm{~V}$ \\
Computational resolution & $n_{x} \times n_{y}$ & $1025 \times 129$ \\
\hline
\end{tabular}

Table 2: Necessary scales and resolution used in the numerical simulation of electro-osmotic flow presented in Section 4. 


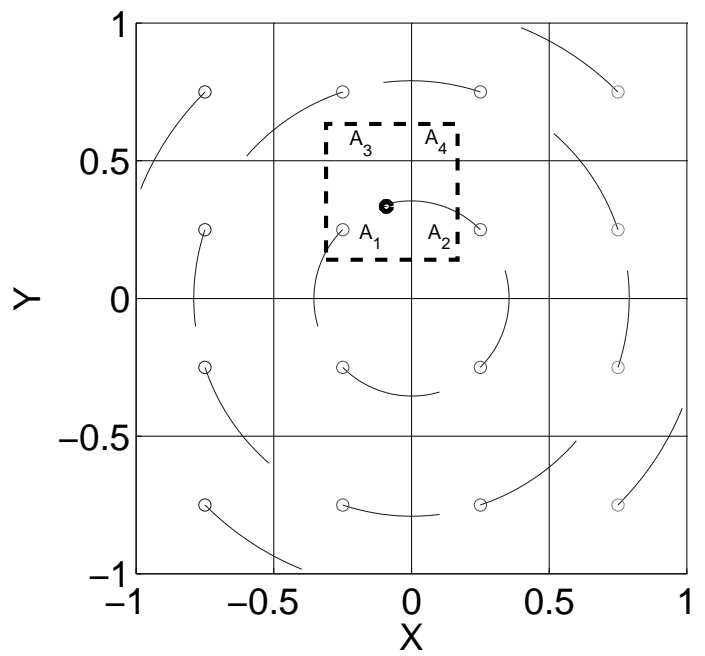

Figure 1: Initially, a fluid parcel occupies a computational cell, and cells are shown with a solid line as the boundary, where the center of a cell is marked by $\circ$. Each cell center $\circ(i, j)$ is the streamline position $s\left(t^{n}\right)$. Solid curves are streamlines that passes through $\circ$, which have been traced by solving (7). For a particular cell, the position $s\left(t^{n+1}\right)$ on the streamline has been marked by $\bullet$, where the associated fluid parcel is shown with a broken line $(---)$. Clearly, this parcel occupies 4 neighbouring cells. 


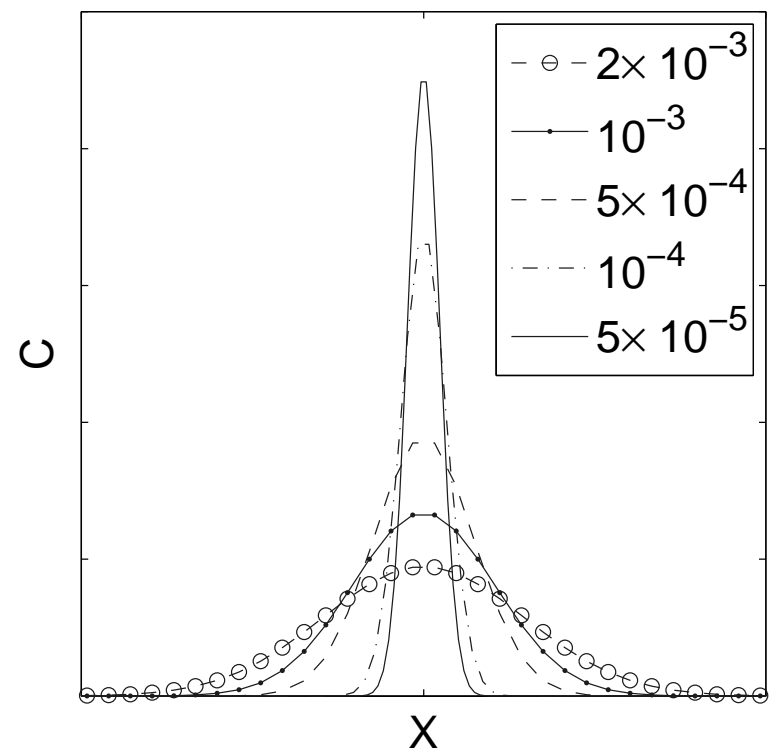

Figure 2: Artificial mass diffusion is examined with a simple 'toy' model, where exact solution is known. The solution, $C(x, T)$, at some fixed time, $t=T$ for varying values of inverse mesh Peclet number, $\frac{1}{P e}: 2 \times 10^{-3}, 10^{-3}, 5 \times 10^{-4}, 10^{-4}, 5 \times 10^{-5}$. As $\frac{1}{P e}$ decreases, the concentration profiles become steeper. Clearly, a smaller value of $P e$ correspond to more spreading or diffusion of mass. This shows that the order of magnitude for the local truncation error may vary by a factor of 30 to 40 , but this small error has a remarkable effect on the finite time mass diffusion. 

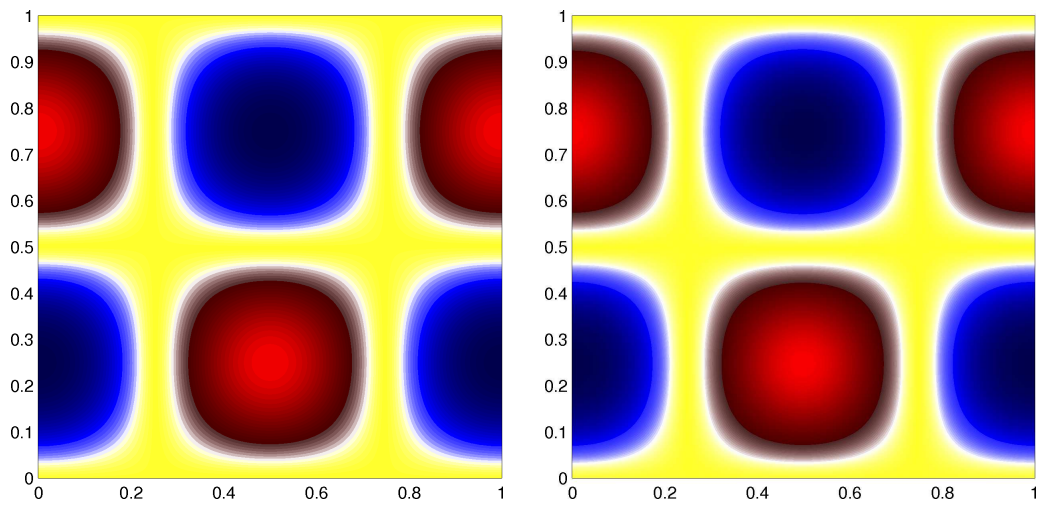

Figure 3: The solution $u(x, y, t)$ of the Taylor-Green flow: (a) numerical solution after 100 time steps, (b) exact solution at the equivalent time, $t$. The numerical solution exhibits a good agreement with the exact solution, verifying the accuracy of the code. The domain has length $x / h=1$ and width $y / h=1$. 


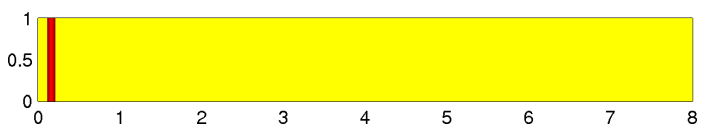

(o) Initial $C(x, y, 0)$
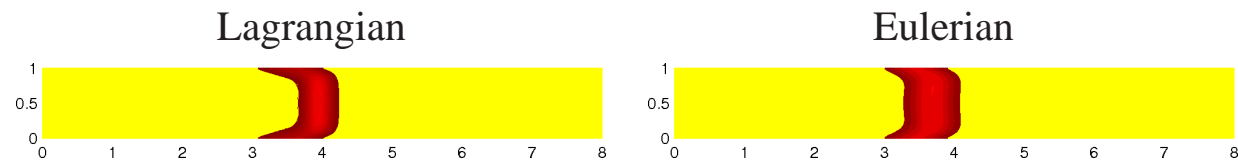

(a) $\frac{1}{P e}=2 \times 10^{-3}$

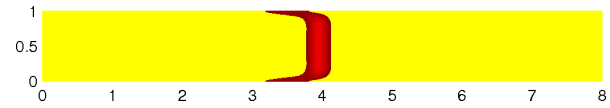

(f) $\frac{1}{P e}=2 \times 10^{-3}$

(b) $\frac{1}{P e}=1 \times 10^{-3}$
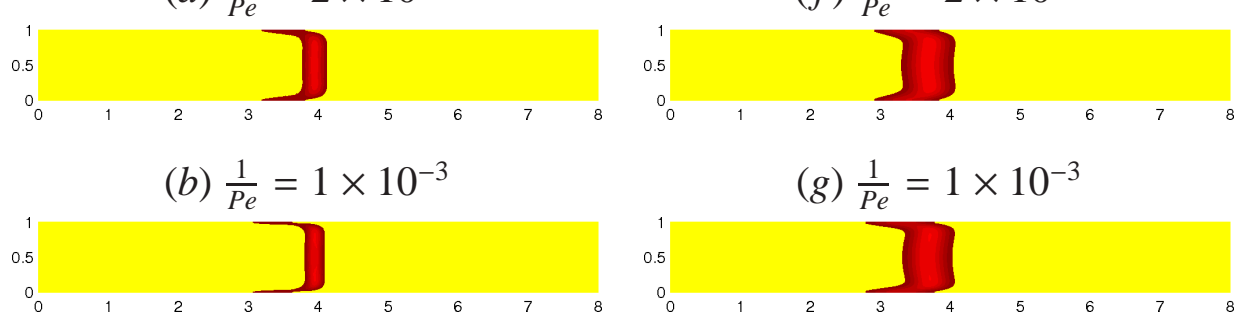

(c) $\frac{1}{P e}=5 \times 10^{-4}$

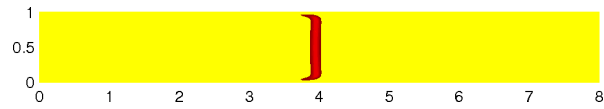

(g) $\frac{1}{P e}=1 \times 10^{-3}$

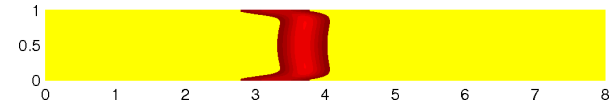

(d) $\frac{1}{P e}=1 \times 10^{-4}$

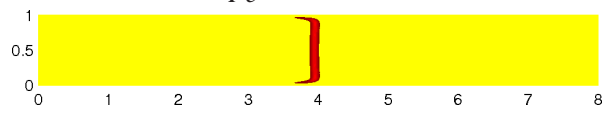

(h) $\frac{1}{P e}=5 \times 10^{-4}$

(e) $\frac{1}{P e}=5 \times 10^{-5}$

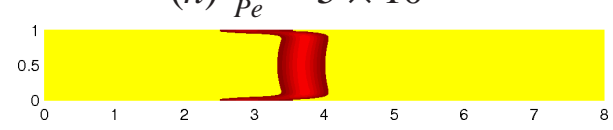

(i) $\frac{1}{P e}=1 \times 10^{-4}$

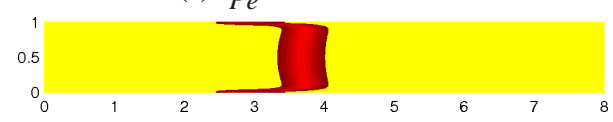

(j) $\frac{1}{P e}=5 \times 10^{-5}$

Figure 4: The effect of $P e$ on the concentration $C(x, y, t)$ at $t=4.4$ in a channel of length $x / h=8$ and width $y / h=1$. (o) Initial concentration field, $C(x, y, 0)$. Simulated $C(x, y, 4.4)$ for various $\frac{1}{P e}$ are shown in subsequent images: (a-e) Lagrangian and $(f-j)$ Eulerian upwind. The values of $\frac{1}{P e}$ are chosen as follows. $(a, f) 2 \times 10^{-3},(b, g) 1 \times 10^{-3},(c, h) 5 \times 10^{-4},(d, i) 1 \times 10^{-4}$, and $(e, j) 5 \times 10^{-5}$. Clearly, we see that the Lagrangian method has resolved the effect of diffusion coefficient. The Eulerian method is plagued with artificial numerical diffusion. 


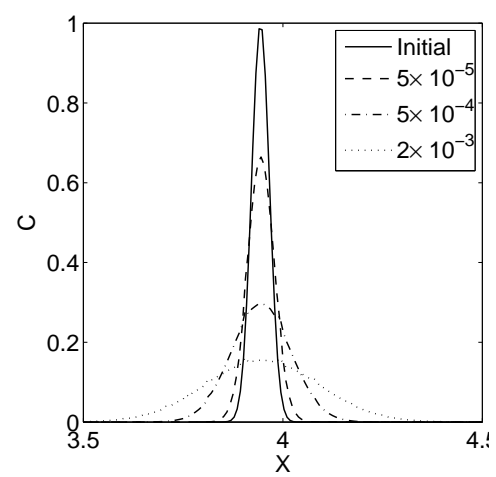

(a)

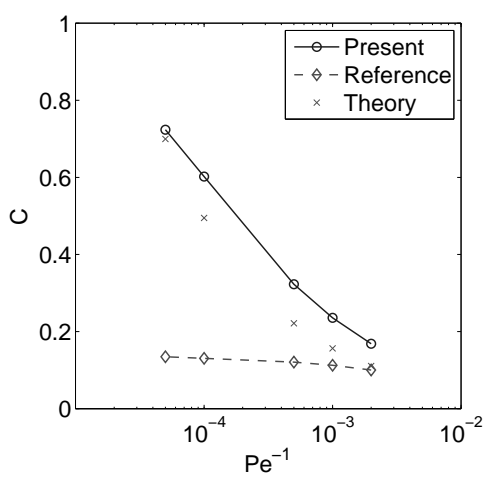

(b)

Figure 5: (a) Profiles of $C(x, 0.5,4.4)$ for $\frac{1}{P e}=2 \times 10^{-3}, 5 \times 10^{-4}, 5 \times 10^{-5}$, and the initial, $C(x, 0.5,0)$, which has been translated for comparison purposes. As expected, distribution of $C$ widens and its maximum value decreases if $P e$ increases. (b) Corresponding to the results in Fig. 4, the maximum values of $C$ for increasing $P e$ are shown. Comparison of the results from the present model and the reference model shows that the present result agrees well with the theoretical prediction. 


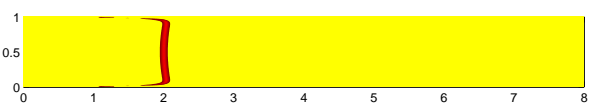

(a)

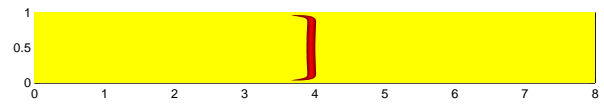

(b)

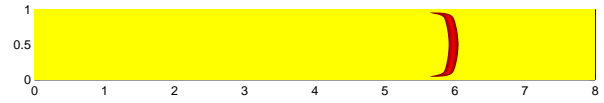

$(c)$

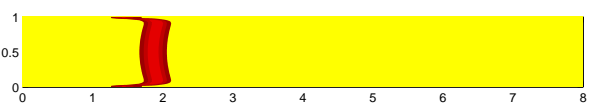

(d)

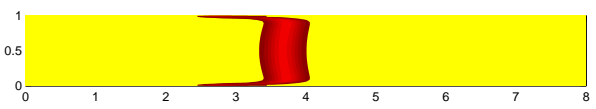

(e)

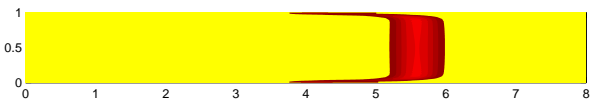

$(f)$

Figure 6: Concentration profiles for $\frac{1}{P e}=5 \times 10^{-5}$ at (a)/(d) $t=2.2$, (b)/(e) $t=4.4$, and (c)/(f) $t=6.6$ in a channel of length $x / h=8$ and width $y / h=1$. Figures (a), (b), and (c) were calculated using a Lagrangian approach for the advection term, while figures (d), (e), and (f) were calculated using an upwind scheme for the advection term.

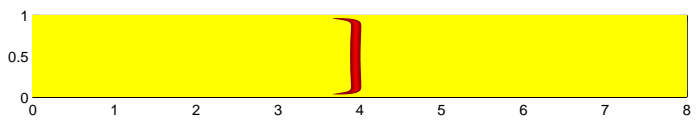

(a)

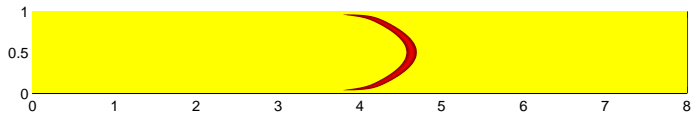

(b)

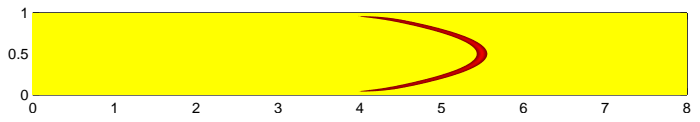

(c)

Figure 7: Combined effects of electro-osmotic and hydro-dynamic transport. Concentration fields are simulated with the Lagrangian model for $\frac{1}{P e}=5 \times 10^{-5}$ with increasing pressure values at the input: (a) $P_{0}=0.048 \mathrm{~Pa}$, (b) $P_{0}=0.24 \mathrm{~Pa}$, and (c) $P_{0}=0.48 \mathrm{~Pa}$. The rectangular sample plug takes a parabolic shape if the input pressure increases. 